\title{
Subcutaneous immunoglobulin replacement therapy in the treatment of patients with primary immunodeficiency disease
}

This article was published in the following Dove Press journal:

Therapeutics and Clinical Risk Management

16 December 2009

Number of times this article has been viewed

\section{Suzanne Skoda-Smith \\ Troy R Torgerson \\ Hans D Ochs}

Seattle Children's Research Institute and Department of Pediatrics, University of Washington, Seattle, Washington
Correspondence: Hans D Ochs Seattle Children's Research Institute and Department of Pediatrics, University of Washington, 1900 Ninth Ave., C9S-7, Seattle,WA 9810।, USA

Tel + I 2069877318

Fax + I 2069877310

Email hans.ochs@seattlechildrens.org

\begin{abstract}
Antibody deficiency is the most frequently encountered primary immunodeficiency disease (PIDD) and patients who lack the ability to make functional immunoglobulin require life-long replacement therapy to prevent serious bacterial infections. Human serum immunoglobulin manufactured from pools of donated plasma can be administered intramuscularly, intravenously or subcutaneously. With the advent of well-tolerated preparations of intravenous immunoglobulin (IVIg) in the 1980s, the suboptimal painful intramuscular route of administration is no longer used. However, some patients continued to experience unacceptable adverse reactions to the intravenous preparations, and for others, vascular access remained problematic. Subcutaneously administered immunoglobulin ( $\mathrm{SCIg}$ ) provided an alternative delivery method to patients experiencing difficulties with IVIg. By 2006, immunoglobulin preparations designed exclusively for subcutaneous administration became available. They are therapeutically equivalent to intravenous preparations and offer patients the additional flexibility for the self-administration of their product at home. SCIg as replacement therapy for patients with primary antibody deficiencies is a safe and efficacious method to prevent serious bacterial infections, while maximizing patient satisfaction and improving quality of life.
\end{abstract}

Keywords: subcutaneous immunoglobulin, primary immunodeficiency disease, antibody deficiency, X-linked agammaglobulinemia, common variable immune deficiency

\section{Background}

The primary immunodeficiency diseases (PIDD) are a heterogeneous group of inherited disorders that affect the cells and proteins of the immune system. Defects in antibody production are the most common type of PIDD comprising about $60 \%$ of the primary immunodeficiencies encountered in practice. Prior to the advent of antibiotics, patients with antibody deficiency succumbed early in life to serious bacterial infections such as pneumonia. In 1952, the first literature report of a patient with recurrent sinopulmonary infections, lacking the immunoglobulin fraction in his serum, was published in Pediatrics by Colonel Ogden Bruton. ${ }^{1}$ He reasoned the patient might benefit from replacement human immunoglobulin, since the gamma fraction contained the antibodies known to combat bacterial infections. As a result of the blood banking and processing advances made during World War II, immunoglobulin fractionated from human plasma was available due to the efforts of Edwin Cohn, who employed a cold-alcohol fractionation process to separate plasma proteins. $^{2}$ Although his work was initially focused on using the albumin fraction on the battlefield as a blood substitute, attention later turned to Cohn fraction II which contained human antibody. submit your manuscript | www.dovepress.con

Dovepress 
The syndrome described by Bruton would come to be known as X-linked agammaglobulinemia (XLA), a defect in the development of antibody producing $\mathrm{B}$ lymphocytes of boys with a mutation in the Bruton tyrosine kinase (BTK) gene. With adequate, regularly scheduled immunoglobulin replacement, using products that still employed Cohn's fractionation process as one of the preparative steps, the first generation of boys with this disease are living into their third and fourth decades and beyond without endorgan damage from chronic, recurrent bacterial infections. Lifelong antibody replacement therapy is also indicated for common variable immune deficiency (CVID), a defect of antibody production often with later onset. Many other primary immune defects have a component of antibody deficiency and also benefit from replacement with human immunoglobulin.

\section{The evolution of immunoglobulin replacement therapy}

Following Bruton's initial description of the beneficial effects of antibody replacement in a patient with severe deficiency in immunoglobulin production, intramuscular dosing became the primary route of administration. In the mid 1950s weekly intramuscular immunoglobulin (IMIg) injections became the standard of care, however, they were poorly tolerated by most patients, particularly children, due to the discomfort that accompanied them. The volume of product that could be given was limited, and serum IgG levels rarely approached physiologic concentrations. Compliance with intramuscular injections was understandably poor in many patients. Occasionally, some of the IMIg would inadvertently enter a vein, resulting in a severe anaphylactic reaction.

In an attempt to optimize the response to therapy and to decrease discomfort, some practitioners used whole plasma infusions intravenously. During the 1960s attempts were made to formulate a usable intravenous preparation of immunoglobulin. Early attempts were fraught with problems related to the aggregation of IgG molecules during preparation and storage. These aggregates are thought to activate complement when infused intravenously, producing severe systemic side effects such as hypotension, angioedema, rigors and fever. The first immunoglobulin products introduced for widespread intravenous use were Gamimune ${ }^{\circledR}$ (Cutter Biologicals) and Sandoglobulin ${ }^{\circledR}$ (Sandoz Pharmaceuticals, now Novartis) in the early 1980s. With the addition of sugars, and subsequently amino acids as stabilizers to prevent aggregation of IgG molecules, the systemic side effects associated with IVIg have been markedly reduced in subsequent generations of IVIg products. The currently available products, summarized in Table 1, have mild systemic reaction rates of $2 \%$ to $6 \%$. ${ }^{3}$ While most reactions are related to the rate of infusion, patients are also more likely to experience reactions to IVIg during the first and second infusion, after a significant interruption in therapy, or if they have an active infection at the time of their infusion.

Table I Characteristics of immune globulin products currently available in the United States

\begin{tabular}{|c|c|c|c|c|c|c|}
\hline & Manufacturer & IgG conc. & $\begin{array}{l}\text { IgA conc. } \\
(\mu \mathrm{g} / \mathrm{mL})\end{array}$ & Excipients & $\begin{array}{l}\text { Osmolality } \\
\text { (m0sm/kg) }\end{array}$ & Viral safety \\
\hline \multicolumn{7}{|c|}{ Products intended for intravenous use (liquid preparations) } \\
\hline Flebogamma ${ }^{\circledast}$ DIF & Grifols & $5 \%$ & $<50$ & $50 \mathrm{mg} / \mathrm{mL}$ D-sorbitol & $240-370$ & $\begin{array}{l}\text { Chromatography } \\
\downarrow \mathrm{pH} / \uparrow \text { temp treatment, S/D, NF }\end{array}$ \\
\hline Gammagard Liq. & Baxter & $10 \%$ & 37 & $250 \mathrm{mM}$ glycine & $240-300$ & $\begin{array}{l}\text { Chromatography } \\
\downarrow_{\mathrm{pH} / \uparrow \text { temp treatment, S/D, NF }}\end{array}$ \\
\hline Gamunex $^{\circledR}$ & Talecris & $10 \%$ & 46 & $200 \mathrm{mM}$ glycine & 258 & $\begin{array}{l}\text { Chromatography } \\
\downarrow \text { pH incubation }\end{array}$ \\
\hline Octagam $^{\circledR}$ & Octapharma & $5 \%$ & $<100$ & $10 \%$ maltose & $310-380$ & Chromatography S/D \\
\hline Privigen $^{\circledR}$ & CSL-Behring & $10 \%$ & $<25$ & 250 mM L-proline & 320 & $\begin{array}{l}\text { Chromatography } \\
\downarrow_{\mathrm{pH}} \text { incubation, NF }\end{array}$ \\
\hline \multicolumn{7}{|c|}{ Products intended for subcutaneous use } \\
\hline Vivaglobin $^{\circledR}$ & CSL-Behring & $16 \%$ & $<1700$ & $\begin{array}{l}3 \mathrm{mg} / \mathrm{mL} \mathrm{NaCl} \\
250 \mathrm{mM} \text { glycine }\end{array}$ & 445 & $\begin{array}{l}\text { Chromatography } \\
\uparrow \text { Temp treatment }\end{array}$ \\
\hline \multicolumn{7}{|c|}{ Products intended for intramuscular use } \\
\hline GamaSTAN $^{\circledR}$ & Talecris & $16 \%$ & NL & $300 \mathrm{mM}$ glycine & NL & $S / D$ \\
\hline
\end{tabular}

Abbreviations: NF, nanofiltration; UF, ultrafiltration; S/D, solvent/detergent; NL, not listed. 
Other significant though less frequent side effects reported with IVIg therapy include renal failure, aseptic meningitis and thrombosis. Episodes of renal failure with sucrose containing IVIg products prompted an FDA warning related to the use of sugar containing product with high osmolality in patients with pre-existing renal insufficiency. These side effects are also related to the hydration status of the patient and to higher doses of IVIg. ${ }^{4}$ Finally, there was recognition in the late 1980s and early 1990s, that the accepted preparative approaches that included a single viral inactivation step were not adequate to prevent transmission of, at that time uncharacterized virus, Hepatitis C. Subsequent immunoglobulin products have been manufactured using at least two different viral inactivation procedures, and some newer generation products attempt to screen out prions as well.

\section{Subcutaneous therapy}

The first report of subcutaneous immunoglobulin therapy (SCIg) using an available intramuscular preparation was published in 1980 by Berger and colleagues. Three adult patients with inadequate infection control and poor compliance with IMIg or intolerance of plasma replacement infusions were given $5-15 \mathrm{~mL}$ of immunoglobulin by slow subcutaneous infusion $(1-2 \mathrm{~mL} / \mathrm{h})$ several times a week. ${ }^{5}$ Patients were able to maintain their serum IgG concentrations at $500 \mathrm{mg} / \mathrm{dL}$ or greater, and had marked improvement in their quality of life. One patient was even able to begin to work full time. Soon after this report, IVIg therapy became readily available and clinicians embraced the intravenous method of administration. Higher levels of serum IgG were easily attainable and the clinical benefit was realized with a substantial decrease in serious bacterial infections, recurrent pneumonias, and chronic bronchiectasis. ${ }^{6,7}$ Although several investigators throughout the 1980 s continued to publish articles outlining the benefits of subcutaneous infusions using $16 \%$ intramuscular preparations of immunoglobulin, the time required for these slow infusions, and the ready availability of the new IVIg products made the subcutaneous route of administration less desirable for most patients.

Limitations for some patients using the intravenous route soon became evident. By 1991 a letter to the journal Lancet reported the use of an implantable intravenous device (Port-a-cath ${ }^{\circledR}$ ) in an adult patient who no longer had peripheral venous access for IVIg. ${ }^{8}$ However; many clinicians had less success with the use of indwelling intravenous catheters in immunodeficient patients requiring lifelong immunoglobulin therapy and the increased risk of serious bacterial infections in this population made the approach less appealing. Infants were a particularly difficult patient population when it came to maintaining stable venous access.

As clinicians gained more experience with the early IVIg products during the 1980s and early 1990s, it became apparent that adverse reactions such as headache, fever, chills, myalgias, fatigue, and nausea were problematic for a subgroup of patients, even with slow intravenous infusions. The published prevalence of these reactions varied widely (at times reported as high as $27 \%$ ), prompting the need for premedication with acetaminophen, steroids, and antihistamines in some individuals receiving IVIg. ${ }^{9}$ In an attempt to minimize these systemic adverse events, and to make subcutaneous infusions more appealing to patients in terms of time commitment, Gardulf and colleagues reported the delivery of a $16 \%$ intramuscular immunoglobulin preparation widely available in Sweden, using a rapid subcutaneous infusion technique. ${ }^{10}$ The Swedish group administered over 3000 subcutaneous infusions to 25 adult patients, 15 of whom had reported systemic reactions to IMIg or IVIg, using subcutaneous infusion rates of $17-20 \mathrm{~mL} / \mathrm{h}$. Although the groups were small, the rates of mild systemic reactions such as fatigue, dizziness, chills, or nausea were $0.93 \%$ for subcutaneous infusions, $46.3 \%$ for intravenous infusions, and $22.2 \%$ for intramuscular administration. Eight patients receiving IMIg reported 20 severe systemic reactions (1.1\% of infusions), with one experiencing circulatory collapse. There were no severe reactions in the group receiving subcutaneous infusions, in fact almost two thirds of these infusions were given outside the hospital setting, at home or at work. Virtually all patients receiving subcutaneous therapy reported local site reactions of swelling, erythema, tenderness and induration, but in $67 \%$ of the patients, these reactions lasted less than 24 hours. There were no severe systemic reactions noted with subcutaneous administration of the intramuscular immunoglobulin preparation, even in those patients who had previously reported severe reaction with the intramuscular route.

Gardulf's group also attempted "express" subcutaneous infusions, allowing patients who tolerated the $20 \mathrm{~mL} / \mathrm{hr}$ rapid infusion rate to increase it to $40 \mathrm{~mL} / \mathrm{h} .{ }^{11}$ Patients rated their perceptions of local tissue reactions but the actual degree of swelling or erythema was not measured. The percentages of patients reporting local tissue reactions using a $20 \mathrm{~mL} / \mathrm{h}$ rate versus a $40 \mathrm{~mL} / \mathrm{h}$ rate was no different $(50 \%$ for rapid and $46 \%$ for express infusion rates). Overall, $24 \%$ of those reporting local site reactions reported a more intense reaction when 
using the express rate, while $66 \%$ felt there was no change. Ten percent of patients felt the reaction was less intense.

Rapid subcutaneous infusions were soon evaluated in children and found to be feasible. ${ }^{12-14}$ In the United States, where a concentrated ( $16 \%$ or greater) mercury free, intramuscular product was not as readily available as in Europe, Stiehm and colleagues reported that the available intravenous products ranging in concentration from $10 \%-12 \%$, could be given safely via the subcutaneous route. ${ }^{15}$ In the mid to later part of the 1990s, larger scale European prospective studies began to evaluate the safety, efficacy and cost of using an available $16 \%$ intramuscular preparation compared to intravenous immunoglobulin. It was nearly 10 years, however, before the first human trials were done to evaluate an immunoglobulin preparation specifically formulated for subcutaneous use.

\section{Pharmacokinetics of SClg}

The pharmokinetics of exogenous immunoglobulin varies by route of administration. Intravenous infusions, given in the customary doses of 300-400 mg/kg every 3-4 weeks, produces peak serum IgG levels within 15 minutes of completing the infusion, and takes 48-72 hours to become distributed from the intravascular compartment to the total extracellular fluid. Initially, the intravascular concentration of IgG increases by 200-300 mg/dL for every $100 \mathrm{mg} / \mathrm{kg}$ of IVIg given and with redistribution into the extracellular compartment, serum levels fall by approximately $50 \%{ }^{16}$ After reequilibration, IgG is catabolized, with a half-life of approximately 22 days. The half-life of $\mathrm{IgG}$ can be up to 30 days in patients with hypo- or agammaglobulinemia. In contrast, subcutaneous administration results in a more stable and consistent serum IgG concentration, although therapeutic plasma levels are not reached as quickly. ${ }^{17}$ Early studies demonstrated that maximal plasma concentrations of IgG are not reached until 4-6 days after intramuscular or subcutaneous administration. ${ }^{18}$ At an immunoglobulin dose of $100 \mathrm{mg} / \mathrm{kg} /$ week given subcutaneously, steady state is achieved after six months of therapy; however, if the same dose is administered daily for five consecutive days, steady state can be achieved in a week. ${ }^{19}$ For patients transitioning from IVIg to SCIg, subcutaneous infusions given within the first week of receiving the last IV dose allows the IgG level to remain therapeutic.

These pharmacokinetic differences are reflected in the magnitude of difference observed in peak and trough levels between intravenous versus subcutaneous dosing. In one comparative study, patients receiving intravenous therapy every three weeks had peak and trough levels that spanned a range of $900 \mathrm{mg} / \mathrm{dL}$, while the range seen with weekly subcutaneous administration was less than $100 \mathrm{mg} / \mathrm{dL} .{ }^{17}$ Thus, while trough determinations are important guides for assessing the adequacy of IVIg replacement therapy, IgG levels can be assessed at any time after steady state is reached to determine the adequacy of subcutaneous replacement therapy.

Systemic side effects of immunoglobulin treatment have been associated with the significant fluctuations between peak and trough levels with IV infusions. A patient survey in 2008 by the Immune Deficiency Foundation reported that $68 \%$ of respondents noted fatigue and increased susceptibility to infections at the time of their trough nadir just prior to their next IVIg infusion. ${ }^{20}$ Clinicians have also attempted to correlate infusion related side effects such as headache and back pain to high peak serum IgG levels. ${ }^{21}$ In contrast, a lower systemic side effect profile has been consistently observed throughout many reports of subcutaneous replacement therapy, most likely related to the more stable physiologic IgG levels achieved. ${ }^{22}$

The bioavailability of subcutaneous immunoglobulin has not been clearly determined, and this prompted discussion of appropriate dosing when the first North American clinical trial to evaluate a new SCIg (Vivaglobin ${ }^{\circledR}$ ) was designed. The European trial evaluating the same product used doses comparable to the standard IVIg replacement dose, adjusted to provide a comparable weekly amount. (An IVIg dose of $300 \mathrm{mg} / \mathrm{kg}$ every three weeks translated to $100 \mathrm{mg} / \mathrm{kg}$ per week of SCIg). For the North American trial, the Food and Drug Administration (FDA) required a dose determined by a time-averaged area under the curve that was comparable to that seen with IVIg. Based on pharmacokinetics, patients in this trial received an average of 1.37 times the IV dose when converting to subcutaneous product. Mean "trough" levels rose by $39 \%$ (from $786 \mathrm{mg} / \mathrm{dL}$ while on IVIg to $1040 \mathrm{mg} / \mathrm{dL}$ while on SCIg) with conversion from IV to subcutaneous immunoglobulin. ${ }^{23}$ Another European trial published six years before the Vivaglobin ${ }^{\circledR}$ study, evaluating the safety and efficacy of IVIg versus an intramuscular immunoglobulin product given subcutaneously, noted similar serum IgG levels in their patients without an increased dose of immunoglobulin for the subcutaneous arm of the study. Although it was not an endpoint in their study, and only half of the patients had IgG trough levels compared during the first six months of this two-year study, the authors reported median IgG trough levels of $780-840 \mathrm{mg} / \mathrm{dL}$ in patients receiving IVIg and 800-910 mg/dL in patients receiving subcutaneous 
infusions using comparable doses of each product. ${ }^{24}$ The results of the European Vivaglobin ${ }^{\circledR}$ trial confirmed the suggestion by the earlier European trial and no drop in $\operatorname{IgG}$ level was seen using the same cumulative monthly dose for subcutaneous therapy that was given for IV therapy. A sample calculation for directly converting a patient from IVIg to SCIg at the same monthly dose is shown in Table 2.

\section{Efficacy of subcutaneous immunoglobulin}

Prior to the prospective North American and European clinical trials published in 2006 confirming the safety and efficacy of a new $16 \%$ product (Vivaglobin ${ }^{\circledR}$ ) designed only for subcutaneous use, a trial by Chapel and colleagues compared the efficacy and safety of subcutaneous Gammaglobulin (a 16\% intramuscular preparation) to intravenous Endobulin (a $5 \%$ intravenous preparation). This was a randomized crossover trial with a primary endpoint of number and severity of infections during the two-year trial period. Thirty patients were enrolled and randomized with the finding that there was no difference in the efficacy or adverse reaction rate of the two therapies. Mean infection scores for moderate and major infections were the same for both treatments. ${ }^{24}$ This trial excluded patients with high levels of anti-IgA antibodies or a history of severe adverse reactions to a blood product within the last two years, which possibly affected the adverse reaction data. In addition, local site reactions were included with mild systemic reactions in the evaluation of adverse reactions, while most investigators differentiate between systemic reactions and local infusion site reactions of swelling, pain or erythema. The prospective, longitudinal European Vivaglobin ${ }^{\circledR}$ trial evaluated 60 patients with a variety of PIDD, including IgG subclass deficiency, although the majority carried diagnoses of CVID or XLA. Eligible patients could be on subcutaneous immunoglobulin using an intramuscular preparation or IVIg as long as they had been on therapy for at least six months with defined, stable IgG trough levels. No increase in dose was given when transitioning from IVIg therapy. Mean serum IgG trough levels while on Vivaglobin ${ }^{\circledR}$ were analyzed separately for children and adults; however, both groups demonstrated noninferiority when compared with the IVIg preparation. One bacterial pneumonia was reported and the annual rate of serious bacterial infections in the group receiving Vivaglobin ${ }^{\circledR}$ was 0.04 per patient per year. ${ }^{25}$ The North American Vivaglobin ${ }^{\circledR}$ trial, also published in 2006, required a diagnosis of either CVID or XLA for enrollment. Patients with normal levels of serum IgG who lacked evidence of functional antibodies or patients with IgG subclass deficiency were not included in this trial. All 68 enrolled patients were on IVIg at the start of the study which included a baseline observation of 3-4 weeks while the patients continued to receive IVIg, followed by a 12 -week wash in/wash out period after transitioning to an adjusted, higher dose of Vivaglobin ${ }^{\circledR}(1.37 \times$ IVIg dose $)$. The subsequent efficacy phase lasted 52 weeks and the primary endpoint measured was the number of serious bacterial infections

Table 2 Sample calculation process for converting from IVlg to SClg

\begin{tabular}{|c|c|c|}
\hline Step & Calculations & Sample calculation \\
\hline \multirow{4}{*}{1} & \multirow{2}{*}{ Calculation of weekly IVIg dose: } & A $50 \mathrm{~kg}$ patient receives \\
\hline & & 20 grams $(400 \mathrm{mg} / \mathrm{kg})$ of IVIG \\
\hline & \multirow{2}{*}{$\overline{\text { Grams/month }} \div 4=-$ Grams/week } & every four weeks. \\
\hline & & Weekly dose $=20 \div 4=5$ grams $/$ week. \\
\hline \multirow[t]{2}{*}{2} & Calculation of weekly SClg volume (16\% preparation $\left.{ }^{* *}\right)$ : & \\
\hline & Grams/week $_{\text {Gro_-_-_ }} \div 0.16($ Grams $/ \mathrm{mL})=$ & $5 \div 0.16=31.25 \mathrm{~mL} /$ week \\
\hline \multirow[t]{3}{*}{3} & Infuse into 1 or more sites according to these rules: & Based on available Vivaglobin ${ }^{\circledR}$ \\
\hline & $\begin{array}{l}\text { - No more than } 25 \mathrm{~mL} / \mathrm{site} \text { in adults or } 15 \mathrm{~mL} / \text { site in children. } \\
\text { - Infusion rate of no more than } 1 \mathrm{~mL} / 1 \text { minute. }\end{array}$ & $\begin{array}{l}\text { vial sizes of } 3,10 \text {, and } 20 \mathrm{~mL}^{* *} \text {, } \\
\text { round dose up to } 33 \mathrm{~mL} / \text { week. }\end{array}$ \\
\hline & & Infuse in 2 sites once weekly. \\
\hline
\end{tabular}

Notes: **Prescribe according to available vial sizes. $16 \%$ Vivaglobin $^{\circledast}$ is available in single use vials of $3 \mathrm{~mL}(0.48 \mathrm{~g}), 10 \mathrm{~mL}(1.6 \mathrm{~g})$ and $20 \mathrm{~mL}(3.2 \mathrm{~g})$. 
such as pneumonia, meningitis, sepsis, osteomyelitis or visceral abscess during this period. The annual rate of serious bacterial infections during the efficacy phase was the same as in the European trial, and the annual rate of any type of infection was 4.43 per patient per year. ${ }^{23}$ Both trials concluded that Vivaglobin ${ }^{\circledR}$ administered subcutaneously was an effective alternative to IVIg in patients with PIDD.

\section{Safety of subcutaneous immunoglobulin}

Prior to the development of an immunoglobulin product intended only for subcutaneous use, intramuscular or intravenous immunoglobulin preparations were given via the subcutaneous route chiefly because of its lower side effect profile. A multiparameter comparison of IVIg and SCIg is provided in Table 3. From 1991 to 2003, several studies found a low systemic reaction rate ranging from $0.3 \%$ to $3.3 \%$, while local tissue reactions were reported in $2.1 \%$ to $20 \%$ of patients. ${ }^{26}$ The first Scandinavian retrospective study published in 1995 evaluating over 33,000 subcutaneous infusions in patients with PIDD, reported 100 mild and six moderate systemic reactions, and no severe or anaphylactoid reactions. ${ }^{27} \mathrm{Up}$ to $87 \%$ of patients reported local tissue reactions at least once, and this was inversely correlated with their body mass index. Leaner patients were more likely to perceive inconvenience based on local reactions of swelling, tenderness and induration. There were no reported differences with slow versus rapid subcutaneous infusion, other than more pronounced itching associated with rapid infusions. No infusion site abscesses or skin infections were noted. The prospective European Vivaglobin ${ }^{\circledR}$ trial reported systemic adverse reactions in $1 \%$ of infusions. ${ }^{25}$ These consisted chiefly of fever, dizziness, malaise, and nongeneralized skin reactions. There was one report of wheezing that subsided after the infusion was stopped. However, this patient was able to resume the subcutaneous infusion with no further adverse event. One patient developed hypotension 10 minutes after insertion of the subcutaneous needle for his first infusion, and with the infusion of $0.5 \mathrm{~mL}$ of immunoglobulin. This reaction was determined to be a vasovagal response. Transient localized tissue reactions were reported in $28 \%$ of infusions. Patients transitioning from IVIg to Vivaglobin ${ }^{\circledR}$ were most affected, although these local reactions decreased over time.

In the prospective North American Vivaglobin ${ }^{\circledR}$ trial, the most frequently reported treatment related adverse events were headache, nausea, rash, weakness and gastrointestinal symptoms. While the rate of infusion related headache was $1.6 \%$, all other frequently reported events each occurred at a rate of less than $1 \% .{ }^{23}$ Transient localized tissue reactions were reported in $49 \%$ of infusions. One possible explanation for the difference in reported localized reactions between the two trials is that all patients in the US study transitioned from IVIg to the subcutaneous preparation unlike the European study where some patients were already on subcutaneous therapy with another product at the start of the trial.

A recent Italian study suggests there may be a small subset of patients with CVID who deserve special consideration when transitioning from IVIg to subcutaneous therapy because of unacceptable adverse events. Among the 262 patients diagnosed with CVID from 1999 to 2007 , and enrolled in a prospective study by the Italian Primary Immunodeficiency Network, 13 patients developed severe adverse reactions to IVIg (hypotension, chills and fever, headache, nausea, and angioedema) that were not completely prevented by premedication or changing

Table 3 Comparison of SClg and IVIg

\begin{tabular}{lll}
\hline Pharmacokinetics & SCIg & IVIg \\
\hline Efficacy & Consistent serum IgG levels & Wide difference in serum IgG level between peak \\
& Two prospective trials demonstrate & Longh clinical experience demonstrating efficacy \\
noninferiority compared to IVIg & Common \\
Infusion site reactions & Infrequent & Infrequent \\
Factors contributing & Self administered at home. US trials of vivaglobin & Typically administered in an infusion center with \\
to total cost & suggested using higher dose (I.37x) than IVIg & nursing support. \\
Patient satisfaction & Offers flexibility of infusion frequency, site, etc. & Often a better option for patients who have difficulty \\
& Multiple studies confirm enhanced quality of life & with needles and/or self-injection. Preferable in \\
in PIDD patients. & patients who have difficulty with compliance.
\end{tabular}

Abbreviations: Ig, immunoglobulin, IVIg, intravenous immunoglobulin, $\mathrm{SClg}$, subcutaneous immunoglobulin; PIDD, primary immunodeficiency disease. 
to different commercial preparations of IVIg. Only one of these patients had detectable anti-IgA antibodies, which have been proposed as a possible risk factor for severe infusion reactions. All thirteen patients were eventually able to transition to a commercially available subcutaneous product, but two patients experienced adverse reactions with SCIg: one developed hypotension and angioedema with the first infusion of Vivaglobin ${ }^{\circledR}$ given after IVIg infusions had been discontinued for seven years. Subcutaneous infusions were resumed one month later and tolerated well using an alternate subcutaneous product (Subcuvia, Baxter). The second patient developed hypotension, chills and fever after nine months of tolerating subcutaneous infusions of Vivaglobin ${ }^{\circledR}$. His subcutaneous therapy was continued, but at a lower dose, and he was eventually able to gradually increase his dose back to therapeutic levels. ${ }^{28}$ The mechanisms of these single episodes of adverse events are unknown. Importantly, both patients eventually tolerated subcutaneous immunoglobulin infusions demonstrating that SCIg replacement therapy provides a safe alternative that is well tolerated.

Finally, there are special groups of patients, often excluded from clinical trials, who have safely received subcutaneous immunoglobulin therapy. Patients with anti-IgA antibodies, intolerant of low-IgA IVIg have successfully been transitioned to subcutaneous therapy. ${ }^{20,29}$ Young children and pregnant women with PIDD have safely received subcutaneous infusions of immunoglobulin. ${ }^{30,31}$ And while little data exists on the use of subcutaneous immunoglobulin therapy in patients with significant thrombocytopenia, anticoagulant use or bleeding disorders, there is a report of the safe use of subcutaneous therapy in a child with CVID and von Willebrand disease. ${ }^{32}$

\section{Comparative costs of immunoglobulin therapy}

There is no systematic body of data with which to evaluate the costs of intravenous versus subcutaneous immunoglobulin therapy and the implications for health care policy. Since health care economics vary markedly among countries, an analysis of this subject would have to be nation-specific.

Swedish estimates in 1995 comparing the cost of subcutaneous versus intravenous immunoglobulin therapy calculated a reduction of about $\$ 10,000$ per patient per year using the subcutaneous route. This estimate was based on US dollars at 1993 prices. ${ }^{27}$ At the time, the cost of the intramuscular product used for subcutaneous therapy in Sweden was less than the cost of the intravenous product. However, all cost estimates agree that the immunoglobulin product itself constitutes most of the treatment cost, and that home therapy is preferable to institutional infusion therapy, whether the intravenous or subcutaneous route is used. The subcutaneous route greatly facilitates home therapy. Berger estimated a savings in 2004 of \$2000 to \$5000 per patient per year by avoiding facility costs associated with infusions. ${ }^{26}$

Radinsky and Bonagura published a price comparison of associated costs, exclusive of product cost, in New York in 2003 for home subcutaneous versus intravenous immunoglobulin therapy using a home care company. Intravenous therapy cost 3.4 to 6.5 times more in nursing care and equipment compared to subcutaneous therapy. ${ }^{33}$ This was in line with the Swedish finding ten years earlier that home intravenous therapy was three to four times more expensive than home subcutaneous infusions. ${ }^{27} \mathrm{~A} 2005$ cost analysis in Germany estimated that converting $60 \%$ of patients on IVIg to subcutaneous therapy would realize a savings of $€ 17$ to 77 million per year. ${ }^{34}$

No study has taken into account the economic cost of missed hours from work or school, loss of productivity from untreated PIDD, additional costs of antibiotics or acute medical care, or the effect of the pricing structure of immunoglobulin on health care macroeconomics.

\section{Patient satisfaction}

Although the initial Vivaglobin ${ }^{\circledR}$ trials called for a weekly infusion of subcutaneous immunoglobulin, experienced clinicians have discovered that patients do well when they are able to determine their own home infusion schedules. Subcutaneous therapy gives them a large degree of freedom to do this. The volume of immunoglobulin administered per site can range from 5-30 mL, with most clinicians preferring 10-20 $\mathrm{mL}$ per site when using a pump. This can be adjusted according to the body habitus, age, and preference of the patient. Injection locations commonly used include the abdomen, lateral thighs, buttocks and upper arms. One or multiple needle sites per infusion can be used. Tubing sets are now available that make it possible for patients to infuse into four sites simultaneously. The time that it takes to infuse generally varies between one to two hours, but some patients prefer a longer infusion while they are sleeping or performing other activities. Other patients prefer to utilize a slow push method (1 $\mathrm{mL} /$ minute) to infuse a smaller amount $(5-10 \mathrm{~mL} / \mathrm{site})$ of immunoglobulin daily from a hand held syringe. The required weekly dose can be divided in many fashions, as long as the prescribed weekly amount of immunoglobulin is given. The equipment needed is simple: some patients use a 
23 gauge butterfly needle while others prefer a subcutaneous needle that can be loaded into a penlike selfinjector. If an infusion pump is used, only a simple pump is needed - in fact, patients can become discouraged if the pump supplied to them is too complex.

The first quality of life survey related to subcutaneous administration of immunoglobulin in patients with PIDD was performed in Sweden and published in 1993. Gardulf's report on the "life situations" of people with antibody deficiency provides a stark assessment of untreated patients. She used validated tests to assess patient's perceptions of infections, social interaction, and self-rated health status while comparing antibody deficient patients prior to starting immunoglobulin replacement therapy to other defined comparison groups. The eight reference groups included cohorts of patients with other chronic diseases, as well as healthy individuals. The PIDD group that did not receive immunoglobulin treatment prior to entering the study $(\mathrm{n}=10)$ rated their health status similar to patients in renal failure or with rheumatoid arthritis. After 18 months of weekly subcutaneous infusions of $\operatorname{IgG}$, the patients $(n=25)$ reported increased health-related function and improved self-rated health. ${ }^{35}$ From 2004 to 2007 a number of studies outlined the improvement in health-related quality of life for patients receiving subcutaneous immunoglobulin therapy. ${ }^{21,36-39}$ While some of the perceived improvement may be due to the ability to receive therapy easily at home and avoid repeated visits to health care facilities, all studies show that subcutaneous therapy improves a patient's and family's sense of independence and self mastery of their situation. Flexibility of the treatment regimen helps patients cope with this life long therapy.

Clinicians stress that an involved and motivated patient is the ideal candidate for subcutaneous therapy, and some physicians have noted falling IgG levels on home subcutaneous therapy due to waning patient compliance. ${ }^{40}$ Some parents may feel overwhelmed having to perform regular subcutaneous injections for their young child. ${ }^{13}$ Not all people prefer subcutaneous therapy when given a choice. The patients in Chapel's cross-over study preferred IVIg to subcutaneous therapy, $53 \%-33 \%$. The remainder had no preference. ${ }^{24}$ The most frequent reason for dissatisfaction, even in patients who preferred subcutaneous therapy, was the higher infusion frequency. ${ }^{14}$ Still others dislike the local site reactions, which tend to improve over time. Several of the patients who dropped out of the prospective Vivaglobin ${ }^{\circledR}$ trials did so because of dissatisfaction with local reactions. ${ }^{23,25}$ The medical community is trying to address the concerns related to frequency of infusion. A recent publication reported stable serum IgG levels with every second week subcutaneous administration. ${ }^{41} \mathrm{~A}$ trial of locally injected human recombinant hyaluronidase followed by subcutaneous infusion of a full month's volume of immunoglobulin into the same site is being planned in an attempt to stretch infusions to a monthly schedule.

\section{Conclusion}

Subcutaneous immunoglobulin therapy is a safe and effective choice for IgG replacement in patients with PIDD. Two prospective studies demonstrated that it has equivalent efficacy to IVIg in preventing serious bacterial infections in patients with antibody deficiency. The systemic side effect profile is low, and while local tissue reactions are frequent they are often mild and tend to improve over time. Pharmacokinetic studies reveal a more physiologic profile, without large variations in peak and trough levels of serum IgG. A number of studies document improved health-related quality of life in patients on home subcutaneous immunoglobulin therapy. Since the treatment is given at home, using simple supplies and no nursing care, the cost is decreased although most of the cost related to immunoglobulin replacement therapy is due to the cost of the product itself.

Patients reporting less satisfaction with subcutaneous therapy cite the frequency of injections and local site reactions as the primary reasons. However, the method of delivery, volume injected, rapidity of infusion, number of sites, and number of infusions per week can be adjusted to meet specific patient needs as long as the prescribed weekly amount of immunoglobulin is administered. Patients that benefit from this form of therapy include those with poor venous access, those experiencing systemic adverse reactions to IVIg, and patients with anti-IgA antibodies thought to be a risk factor for systemic reactions. In addition, some clinicians have noted more stable $\operatorname{IgG}$ levels in patients with PIDD and protein-losing enteropathy. Subcutaneous therapy has also been utilized in patient groups often excluded in conventional clinical trials, including young children and pregnant women.

The increasing use of the subcutaneous infusion method in patients with PIDD has brought the story of immunoglobulin replacement therapy full circle, since that was the initial method of infusion Bruton utilized in the fifties to treat his first agammaglobulinemic patient. ${ }^{1}$ This treatment has demonstrated efficacy in improving the quality of life and health in a group of patients with an 
otherwise life-threatening chronic disease. With current efforts to improve the effectiveness of health care delivery, it could become a model of how to rationally modify a costly therapy for a chronic illness in order to decrease cost and enhance health-related quality of life while maintaining efficacy.

\section{Disclosures}

The authors report no conflicts of interest in this work.

\section{References}

1. Bruton OC. Agammaglobulinemia. Pediatrics. 1952;9:722-728.

2. Berger M. A history of immunoglobulin therapy, from the Harvard crash program to monoclonal antibodies. Curr Allergy Asthma Rep. 2002;2:368-378.

3. Orange JS, Hossny EM, Weiler CR, et al. Use of intravenous immunoglobulin in human disease; a review of the evidence by members of the primary immunodeficiency committee of the AAAAI. J Allergy Clin Immunol. 2006;117:S525-S553.

4. Pierce CR, Jain N. Risks associated with the use of intravenous immunoglobulin. Transfus Med Rev. 2003;17:241-254.

5. Berger M, Cupps TR, Fauci AS. Immunoglobulin replacement therapy by slow subcutaneous infusion. Ann Intern Med. 1980;93: 55-56.

6. Cunningham-Rundles C, Siegel FP, Smithwick EM, et al. Efficacy of intravenous immunoglobulin in primary immunodeficiency disease. Ann Intern Med. 1984;101:435-439.

7. Quartier P, Debre M, De Blic J, et al. Early and prolonged intravenous immunoglobulin replacement in childhood agammaglobulinemia: a retrospective survey of 31 patients. J Pediatr. 1999;134: 589-596.

8. Webb DB, Kendra JR, Gross E, Stamatakis JD. Infusion of intravenous immunoglobulin via implantable subcutaneous catheter. Lancet. 1991;337:1617-1618.

9. Lederman HM, Roifman CM, Lavi S, Gelfand EW. Corticosteroids for prevention of adverse reactions to intravenous immune serum globulin infusions in hypogammaglobulinemic patients. Am J Med. 1986;81:443-446.

10. Gardulf A, Hammarstrom L, Edvard Smith CI. Home treatment of hypogammaglobulinemia with subcutaneous gammaglobulin by rapid infusion. Lancet. 1991;338:162-166.

11. Hansen S, Gustafson R, Smith CIE, Gardulf A. Express subcutaneous IgG infusions: decreased time of delivery with maintained safety. Clin Immunol. 2002;104:237-241.

12. Thomas MJ, Brennan VM, Chapel HH. Rapid subcutaneous immunoglobulin infusions in children. Lancet. 1993;342:1432-1433.

13. Abrahamsen TG, Sandersen H, Bustnes A. Home therapy with subcutaneous immunoglobulin infusions in children with congenital immunodeficiencies. Pediatrics. 1996;98:1127-1131.

14. Gaspar J, Gerritsen B, Jones A. Immunoglobulin replacement treatment by rapid subcutaneous infusion. Arch Dis Child. 1998;79:48-51.

15. Stiehm ER, Casillas AM, Finkelstein JZ, et al. Slow subcutaneous human intravenous immunoglobulin in the treatment of antibody immunodeficiency: use of an old method with a new product. $J$ Allergy Clin Immunol. 1998;101:848-849.

16. Mankarous M, Lee M, Fischer S, Pyun KH, Ochs HD, Oxelius VA, Wedgwood RJ. The half-lives of IgG subclasses and specific antibodies in patients with primary immunodeficiency who are receiving intravenously administered immunoglobulin. J Lab Clin Med. 1988:112:634-640.

17. Gardulf A. Immunoglobulin treatment for primary antibody deficiencies, advantages of the subcutaneous route. Biodrugs. 2007;21:105-116.
18. Smith GN, Griffiths B, Mollison D, Mollison PL. Uptake of IgG after intramuscular and subcutaneous injections. Lancet. 1972;1:1208-1212.

19. Bonilla FA. Pharmacokinetics of immunoglobulin administered via intravenous or subcutaneous routes. Immunol Allergy Clin $\mathrm{N} \mathrm{Am}$. 2008;28:803-819.

20. Moore ML, Quinn JM. Subcutaneous immunoglobulin replacement therapy for primary antibody deficiency: advancements into the $21 \mathrm{st}$ century. Ann Allergy Asthma Immunol. 2008;101:114-121.

21. Gardulf A. Nicolay U, Math DI, et al. Children and adults with primary antibody deficiencies gain quality of life by subcutaneous IgG infusions at home. J Allergy Clin Immunol. 2004;114:936-942.

22. Grunebaum E, Levy Y, Shoenfeld Y. Novel aspects of hypogammaglobulinemic states: subcutaneous immunoglobulin treatment. Isr Med Assoc J. 2002;4:288-289.

23. Ochs HD, Gupta S, Kiessling P, et al. Safety and efficacy of selfadministered subcutaneous immunoglobulin in patients with primary immunodeficiency disease. J Clin Immunol. 2006;26:265-273.

24. Chapel HM, Spickett GP, Ericson D, et al. The comparison of the efficacy and safety of intravenous versus subcutaneous immunoglobulin replacement therapy. J Clin Immunol. 2000;20:94-100.

25. Gardulf A. Nicolay U, Asensio O, et al. Rapid subcutaneous IgG replacement therapy is effective and safe in children and adults with primary immunodeficiencies - a prospective, multi-national study. J Clin Immunol. 2006;26:177-185.

26. Berger M. Subcutaneous immunoglobulin replacement in primary immunodeficiencies. Clin Immunol. 2004;112:1-7.

27. Gardulf A, Andersen V, Bjorkander J, et al. Subcutaneous immunoglobulin replacement in patients with primary antibody deficiencies: safety and costs. Lancet. 1995;345:365-369.

28. Quinti I, Soresina A, Agostini C, et al. Prospective study on CVID patients with adverse reactions to intravenous or subcutaneous IgG administration. J Clin Immunol. 2008;9169-9199.

29. Sundin U, Nava S, Hammarstrom L. Induction of unresponsiveness against IgA in IgA-deficient patients on subcutaneous immunoglobulin infusion therapy. Clin Exp Immunol. 1998;112:341-346.

30. Fasth A, Nystrom. Safety and efficacy of subcutaneous human immunoglobulin in children with primary immunodeficiency. Acta Paediatrica. 2007;96:1474-1478.

31. Gardulf A, Andersson E, Lindqist M, et al. Rapid subcutaneous IgG replacement therapy at home for pregnant immunodeficient women. J Clin Immunol. 2001;21:150-154.

32. Arora R, Newton TC, Nelson MR. Subcutaneous immunoglobulin therapy in an 11-year-old patient with common variable immunodeficiency and von Willebrand disease. Ann Allergy Asthma Immunol. 2007;99:367-370

33. Radinsky S, Bonagura VR. Subcutaneous immunoglobulin infusion as an alternative to intravenous immunoglobulin. JAllergy Clin Immunol. 2003;112:630-633.

34. Hogy B, Keinecke HO, Borte M. Pharmacoeconomic evaluation of immunoglobulin treatment in patients with antibody deficiencies from the perspective of the German statutory health insurance. Eur J Health Econom. 2005;50:24-29.

35. Gardulf A, Bjorvell H, Gustafson R, et al. The life situations of patients with primary antibody deficiency untreated or treated with subcutaneous immunoglobulin infusions. Clin Exp Immunol. 1993;92:200-204.

36. Nicolay U, Kiessling P, Berger M, et al. Health-related quality of life and treatment satisfaction in North American patients with primary immunedeficiency diseases receiving subcutaneous IgG self-infusions at home. J Clin Immunol. 2006;26:65-72.

37. Gardulf A, Nicolay U. Replacement IgG therapy and self therapy at home improve the health-related quality of life in patients with primary antibody deficiencies. Curr Opin Allergy Clin Immunol. 2006;6:434-442.

38. Gardulf A, Borte M, Ochs HD, et al. Prognostic factors for health-related quality of life in adults and children with primary antibody deficiencies receiving SCIg home therapy. Clin Immunol. 2008;126:81-88. 
39. Fasth A, Nystrom J. Quality of life and health-care resource utilization among children with primary immunodeficiency receiving home treatment with subcutaneous human immunoglobulin. J Clin Immunol. 2008;28:370-378.

40. Dams ETM, van der Meer JWM. Subcutaneous immunoglobulin replacement in patients with primary antibody deficiencies. Lancet. $1995 ; 345: 864$
41. Gustafson R, Gardulf A, Hansen S, et al. Rapid subcutaneous immunoglobulin administration every second week results in high and stable serum immunoglobulin $\mathrm{G}$ levels in patients with primary antibody deficiencies. Clin Exp Immunol. 2008;152:274-279.

\section{Publish your work in this journal}

Therapeutics and Clinical Risk Management is an international, peerreviewed journal of clinical therapeutics and risk management, focusing on concise rapid reporting of clinical studies in all therapeutic areas, outcomes, safety, and programs for the effective, safe, and sustained use of medicines. This journal is indexed on PubMed Central, CAS,
EMBase, Scopus and the Elsevier Bibliographic databases. The manuscript management system is completely online and includes a very quick and fair peer-review system, which is all easy to use. Visit http://www.dovepress.com/testimonials.php to read real quotes from published authors.

Submit your manuscript here: http://www.dovepress.com/therapeutics-and-clinical-risk-management-journal 